\title{
Lymph node ratio-based staging system as an alternative to the current TNM staging system to assess outcome in adenocarcinoma of the esophagogastric junction after surgical resection
}

\author{
Hongdian Zhang ${ }^{1, *}$, Xiaobin Shang ${ }^{1, *}$, Chuangui Chen ${ }^{1}$, Yongyin Gao ${ }^{2}$, Xiangming \\ $X_{i a o^{3}}$, Peng Tang ${ }^{1}$, Xiaofeng Duann ${ }^{1}$, Mingjian Yang ${ }^{1}$, Hongjing Jiang ${ }^{1}$ and Zhentao \\ $\mathbf{Y} \mathbf{u}^{1}$ \\ ${ }^{1}$ Department of Esophageal Cancer, Tianjin Medical University, Cancer Institute and Hospital, Key Laboratory of Cancer \\ Prevention and Therapy of Tianjin City, Tianjin, China \\ ${ }^{2}$ Department of Cardiopulmonary Function, Tianjin Medical University, Cancer Institute and Hospital, Key Laboratory of \\ Cancer Prevention and Therapy of Tianjin City, Tianjin, China \\ ${ }^{3}$ Department of General Surgery, Weifang People's Hospital, Shandong, China \\ * These authors have contributed equally to this work \\ Correspondence to: Zhentao Yu, email: yuzhtao@hotmail.com
}

Keywords: adenocarcinoma of the esophagogastric junction; lymph node metastasis; metastatic lymph node ratio; tumor-Nratio-metastasis (TrNM) staging system; prognosis

Received: March 14, $2016 \quad$ Accepted: July 27, 2016

Published: August 10, 2016

\section{ABSTRACT}

This study aimed to assess the prognostic value of the hypothetical tumor- $\mathrm{N}$-ratio ( $r N$ )-metastasis (TrNM) staging system in adenocarcinoma of the esophagogastric junction (AEG). The clinical data of 387 AEG patients who received surgical resection were retrospectively reviewed. The optimal cut-off point of $\mathrm{rN}$ was calculated by the best cut-off approach using log-rank test. Kaplan-Meier plots and Cox regressions model were applied for univariate and multivariate survival analyses. A TrNM staging system based on $\mathrm{r}$ was proposed. The discriminating ability of each staging was evaluated by using an adjusted hazard ratio (HR) and a $-2 \log$ likelihood. The prediction accuracy of the model was assessed by using the area under the curve (AUC) and the Harrell's C-index. The number of examined lymph nodes (LNs) was correlated with metastatic LNs $(r=0.322, P<0.001)$ but not with $r N(r=0.098, P>$ 0.05 ). The optimal cut-points of $\mathrm{rN}$ were calculated as $0,0 \sim 0.3,0.3 \sim 0.6$, and $0.6 \sim 1.0$. Univariate analysis revealed that $\mathrm{pN}$ and $\mathbf{r N}$ classifications significantly influenced patients' RFS and OS $(P<\mathbf{0 . 0 0 1})$. Multivariate analysis adjusted for significant factors revealed that $\mathrm{rN}$ was recognized as an independent risk factor. A larger $H R$, a smaller -2log likelihood and a larger prediction accuracy were obtained for $\mathrm{rN}$ and the modified TrNM staging system. Taken together, our study demonstrates that the proposed $\mathrm{N}$-ratio-based TrNM staging system is more reliable than the TNM staging system in evaluating prognosis of AEG patients after curative resection.

\section{INTRODUCTION}

The adenocarcinoma of the esophagogastric junction (AEG) is a special clinical disease with different risk factors, unique clinicopathological characteristics and biological behaviors; this condition is characterized by pathologically different tumors developing in the border between the esophageal squamous epithelium and the gastric adenomatous epithelium [1]. Despite the improvements in multimodal treatment strategies in the past decades, the prognosis of AEG remains poor [2].

Lymph node metastasis has been considered as the foremost factor in determining the prognosis of AEG patients [3]. However, the number of involved LNs relies heavily on the number of removed LNs from each patient. If the number of retrieved and examined LNs is small, 
down migration of the $\mathrm{pN}$ stage may occur. Conversely, if the number is large, up migration of the $\mathrm{pN}$ stage may be observed $[4,5]$.

In order to overcome these limitations, a new modified prognostic tool incorporating the ratio between metastatic and dissected LNs has been proposed by several authors, which can more accurately reflect the degree of lymph node metastasis and reduce stage migration $[4,6]$. Although the prognostic significance of $\mathrm{rN}$ in esophageal and gastric cancers has been extensively investigated, this factor in AEG patients has been rarely examined; and whether the $\mathrm{rN}$ classification is more optimal for prognosis than the $\mathrm{pN}$ classification remains unknown $[7,8]$.

Recently, some studies have indicated that the tumor-ratio-metastasis (TrNM) staging system based on $\mathrm{N}$-ratio can be used as an alternative to the traditional TNM staging system for the prognostic evaluation of tumors. However, to the best of our knowledge, the prognostic significance of the hypothetical TrNM staging system has not yet to be formally investigated in AEG. Hence, in the present study, we investigated whether patients with AEG can be classified into meaningful risk categories on the basis of $\mathrm{rN}$. And the prognostic power of the TrNM staging system was evaluated by comparing it with the current TNM staging system for AEG patients after surgical resection.

\section{RESULTS}

\section{Clinicopathological parameters of patients}

The baseline characteristics of the 389 AEG patients who met the inclusion criteria are shown in Table 1. There were 292(75.1\%) males and 97(24.9\%) females. The median age was 62 years, ranging from 22 86 years. The median value of tumor size was 5.0 $\mathrm{cm}$ (range, $0.5 \sim 12.0 \mathrm{~cm}$ ). With respect to surgery, 306 patients underwent curative proximal gastrectomy, 83 cases underwent total gastrectomy. A total of 6791 lymph nodes were picked up and histologically examined, with an average of 17.5 (median 15, range, 4 71) per case. According to histopathological grading, differentiated tumors were observed in $211(54.2 \%)$ patients, and undifferentiated tumors in remaining 178 (45.8\%) patients.

Based on the criteria of the 7th edition of the UICC/ AJCC TNM staging system, 135(34.7\%) patients were classified as pN0, $97(24.9 \%)$ as pN1, $84(21.6 \%)$ as pN2 and $73(18.8 \%)$ as $\mathrm{pN} 3$, respectively. With regarding to TNM staging, 36 patients were at stage I, 125 patients were at stage II, 82 patients were at stage IIIA, 78 patients were at stage IIIB, and 68 were at stage IIIC.

\section{Correlation analysis among metastatic lymph nodes, $r N$, and removed lymph nodes}

As expected, there was a positive correlation between the number of metastatic LNs and removed LNs according to the Spearman's correlation test $(r=0.322, P$ $<0.001$, Figure 1A). However, the rN was not associated with the number of removed LNs $(r=0.098, P>0.05,1 \mathrm{~b})$. Furthermore, the correlation between $\mathrm{rN}$ and the number of metastatic LNs was significant $(r=0.929, P<0.001$, 1c). These results demonstrated that $\mathrm{rN}$ was not influenced by surgery, however, $\mathrm{pN}$ was influenced by surgery.

\section{Re-classification of N-ratio Categories}

To determine the appropriate cut-points of the $\mathrm{rN}$ value that determine the greatest actuarial survival difference among the resulting subgroups in the entire cohort, our analysis was conducted as follows: firstly, patients having no involved LNs $(\mathrm{rN}=0)$ were assigned to one group because it has been well known that their prognosis was significantly better than patients with metastatic LNs [9]. Furthermore, we determined another two appropriate cut-off points for categorizing the $\mathrm{rN}$ to make it comparable with the AJCC $\mathrm{pN}$ classification. The patients were stratified into 10 subgroups based on 0.1 intervals of $\mathrm{rN}$. As there were few cases in the $0.7,0.8$, 0.9 or $1.0 \mathrm{rN}$ subgroups, the 0.7 and 0.8 subgroups, and the 0.9 and 1.0 subgroups were combined. According to the log-rank test, the another two optimal cut-off points 0.3 and 0.6 were chosen for subsequent analysis. Finally, the $\mathrm{rN}$ intervals were categorized as $\mathrm{rN} 0(0 \%), \mathrm{rN} 1$ (1\% 30\%), rN 2 (31\% 60\%) and rN 3 (> 60\%).

Accordingly, a novel TrNM staging was established: 38 patients were at stage I, 125 patients were at stage II, 115 patients were at stage IIIA, 58 patients were at stage IIIB, and 53 patients were at stage IIIC.

\section{Univariate and multivariate survival analysis}

The 5-year RFS and OS rates for the whole group of patients were $25.7 \%, 30.3 \%$, the median RFS and OS time were 31 and 38 months, respectively.

The 5-year RFS and OS curves of patients based on the $\mathrm{pN}$ and $\mathrm{rN}$ classification are shown in Figure 2. The 5-year RFS rates of patients with $\mathrm{pN} 0, \mathrm{pN} 1, \mathrm{pN} 2$ and pN3 were $40.7 \%, 26.8 \%, 17.9 \%$ and $5.5 \%$, respectively $(P$ $<0.001,2 \mathrm{~A}$ ). The 5 -year OS rates of patients with $\mathrm{pN} 0$, $\mathrm{pN} 1, \mathrm{pN} 2$ and $\mathrm{pN} 3$ were $47.7 \%, 31.4 \%, 19.7 \%$ and $7.0 \%$, respectively $(P<0.001,2 \mathrm{~B})$. The 5 -year RFS rates of patients with rN0, rN1, rN2 and rN3 were 40.7\%, 25.9\%, $13.7 \%$ and $1.9 \%$, respectively $(P<0.001,2 \mathrm{C})$. The 5 -year OS rates of patients with $\mathrm{rN} 0, \mathrm{rN} 1, \mathrm{rN} 2$ and $\mathrm{rN} 3$ were 

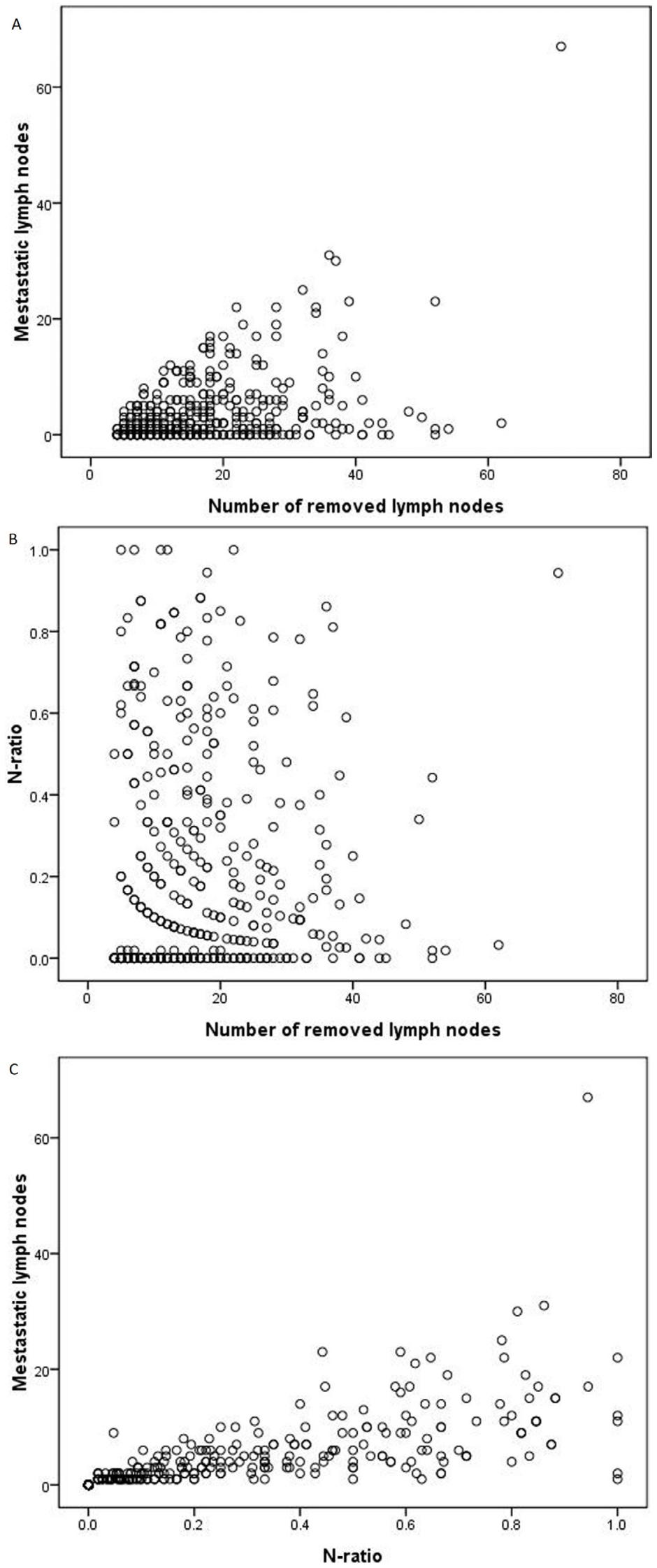

Figure 1: The correlation analysis between (A) the number of removed lymph nodes and metastatic lymph nodes; (B) the number of removed lymph nodes and N-ratio; (C) the number of metastatic lymph nodes and N-ratio. 
Table 1: Univariate survival analysis of clinicopathologic factors associated with RFS and OS in 389 AEG patients after curative surgery

\begin{tabular}{|c|c|c|c|c|c|c|c|}
\hline \multirow{2}{*}{ Clinicopathologic factors } & \multirow{2}{*}{ Cases } & \multicolumn{3}{|c|}{ Recurrence-free survival } & \multicolumn{3}{|c|}{ Overall survival } \\
\hline & & 5-YSR (\%) & $x^{2}$ & $P$ value & 5-YSR (\%) & $x^{2}$ & $P$ value \\
\hline Gender & & & 1.553 & 0.213 & & 2.536 & \\
\hline Male & 292 & 24.7 & & & 28.2 & & 0.111 \\
\hline Female & 97 & 28.9 & & & 36.6 & & \\
\hline Age (years) & & & 0.618 & 0.414 & & 0.678 & \\
\hline$<65$ & 241 & 26.3 & & & 31.8 & & 0.410 \\
\hline$\geq 65$ & 148 & 24.2 & & & 27.8 & & \\
\hline Tumor size $(\mathrm{cm})$ & & & 4.974 & 0.026 & & 4.790 & 0.029 \\
\hline$\leq 5$ & 222 & 28.4 & & & 33.7 & & \\
\hline$>5$ & 167 & 22.2 & & & 25.6 & & \\
\hline Borrmann classification & & & 4.142 & 0.042 & & 6.200 & 0.013 \\
\hline $\mathrm{I}, \mathrm{II}$ & 161 & 31.1 & & & 36.6 & & \\
\hline III, IV & 228 & 21.9 & & & 25.8 & & \\
\hline Laurren type & & & 3.015 & 0.081 & & 4.775 & \begin{tabular}{|l|l|}
0.029 \\
\end{tabular} \\
\hline Intestinal type & 154 & 30.1 & & & 37.3 & & \\
\hline Non-intestinal type & 235 & 20.6 & & & 26.0 & & \\
\hline Histopathological grading & & & 7.741 & 0.005 & & 5.065 & \\
\hline Differentiated & 211 & 31.8 & & & 35.6 & & 0.024 \\
\hline Undifferentiated & 178 & 18.5 & & & 23.6 & & \\
\hline AJCC pT classification & & & 32.686 & 0.000 & & 28.897 & 0.000 \\
\hline pT1 & 10 & 80.0 & & & 90.0 & & \\
\hline pT2 & 48 & 43.7 & & & 49.9 & & \\
\hline pT3 & 26 & 40.3 & & & 43.3 & & \\
\hline pT4 & 305 & 19.7 & & & 24.0 & & \\
\hline AJCC pN classification & & & 68.979 & 0.000 & & 63.895 & 0.000 \\
\hline $\mathrm{pN} 0$ & 135 & 40.7 & & & 47.7 & & \\
\hline pN1 & 97 & 26.8 & & & 31.4 & & \\
\hline $\mathrm{pN} 2$ & 84 & 17.9 & & & 19.7 & & \\
\hline $\mathrm{pN} 3$ & 73 & 5.5 & & & 7.0 & & \\
\hline rN classification & & & 110.179 & 0.000 & & 107.002 & 0.000 \\
\hline rN0 & 135 & 40.7 & & & 47.7 & & \\
\hline rN1 & 127 & 25.9 & & & 30.2 & & \\
\hline rN2 & 73 & 13.7 & & & 17.3 & & \\
\hline rN3 & 54 & 1.9 & & & 2.5 & & \\
\hline
\end{tabular}

AEG, adenocarcinoma of the esophagogastric junction; RFS, recurrence-free survival; OS, overall survival;

5-YSR, 5-year survival rate; $\mathrm{pN}$, lymph node status; rN, N-ratio.

$47.7 \%, 30.2 \%, 17.3 \%$ and $2.5 \%$, respectively $(P<0.001$, 2D).

Univariate and multivariate analyses were applied to evaluate clinicopathological variables relating to 5 -year RFS and OS. The results of univariate analysis are shown in Table 1. For RFS, factors significantly influencing 5 -year RFS were tumor size $(P=0.026)$, Borrmann type $(P=0.042)$, histopathological grading $(P=0.005), \mathrm{pT}$ classification $(P<0.001)$, $\mathrm{pN}$ classification $(P<0.001)$, and $\mathrm{rN}$ classification $(P<0.001)$. For OS, variables significantly associated with 5-year OS were tumor size $(P=0.029)$, Borrmann type $(P=0.013)$, Lauren classification $(P=0.029)$, histopathological grading $(P=$ $0.024)$, pT classification $(P<0.001), \mathrm{pN}$ classification $(P$ $<0.001)$, and $\mathrm{rN}$ classification $(P<0.001)$.

Multiple survival analysis was performed by the Cox's proportional hazard model to identify the independent prognostic factors. We firstly set up a model including all the significant parameters in the univariate analysis as well as $\mathrm{pN}$ classification, except for $\mathrm{rN}$ classification. As a result, pT classification (RFS: $P<$ 0.001; OS: $P=0.001$ ) and $\mathrm{pN}$ classification (RFS: $P<$ 0.001; OS: $P<0.001$ ) were determined to be independent predictors. Similarly, when $\mathrm{pN}$ was substituted by $\mathrm{rN}$ 
Table 2: Multivariate analysis of RFS and OS in 389 AEG patients subjected to curative surgery

\begin{tabular}{|c|c|c|c|c|c|c|}
\hline \multirow[b]{2}{*}{ Clinicopathologic factors } & \multicolumn{2}{|c|}{ Multivariate Analysis 1} & \multicolumn{2}{|c|}{ Multivariate Analysis 2} & \multicolumn{2}{|c|}{ Multivariate Analysis 3} \\
\hline & HR (95\%CI) & $P$ value & HR $(95 \%$ CI $)$ & \begin{tabular}{|l|}
$P$ \\
value
\end{tabular} & HR $(95 \%$ CI $)$ & \begin{tabular}{|l|}
$P$ \\
value
\end{tabular} \\
\hline \multicolumn{7}{|l|}{ Recurrence-free survival } \\
\hline Tumor size & $1.041(0.819 \sim 1.325)$ & 0.741 & $1.043(0.820 \sim 1.326)$ & 0.732 & $1.034(0.812 \sim 1.318)$ & 0.786 \\
\hline Borrmann classification & $1.160(0.912 \sim 1.474)$ & 0.226 & $1.175(0.924 \sim 1.494)$ & 0.188 & $1.175(0.924 \sim 1.495)$ & 0.187 \\
\hline Histopathological grading & $1.157(0.914 \sim 1.464)$ & 0.214 & $1.150(0.928 \sim 1.487)$ & 0.176 & $1.151(0.926 \sim 1.485)$ & 0.175 \\
\hline pT classification & $1.440(1.204 \sim 1.726)$ & 0.000 & $1.401(1.168 \sim 1.680)$ & 0.000 & $1.401(1.168 \sim 1.680)$ & 0.000 \\
\hline pN classification & $1.442(1.293 \sim 1.608)$ & 0.000 & - & - & $1.047(0.869 \sim 1.262)$ & 0.629 \\
\hline $\mathrm{rN}$ classification & - & - & $1.637(1.449 \sim 1.850)$ & 0.000 & $1.573(1.283 \sim 1.929)$ & 0.000 \\
\hline \multicolumn{7}{|l|}{ Overall survival } \\
\hline Tumor size & $1.044(0.815 \sim 1.336)$ & 0.733 & $1.039(0.812 \sim 1.331)$ & 0.759 & $1.031(0.804 \sim 1.322)$ & 0.808 \\
\hline Borrmann classification & $1.244(0.970 \sim 1.596)$ & 0.086 & $1.259(0.981 \sim 1.615)$ & 0.070 & $1.259(0.981 \sim 1.615)$ & 0.070 \\
\hline Lauren type & $1.045(0.803 \sim 1.360)$ & 0.741 & $1.019(0.783 \sim 1.325)$ & 0.891 & $1.016(0.781 \sim 1.322)$ & 0.905 \\
\hline Histopathological grading & $1.063(0.825 \sim 1.370)$ & 0.635 & $1.097(0.852 \sim 1.413)$ & 0.471 & $1.094(0.850 \sim 1.409)$ & 0.486 \\
\hline pT classification & $1.436(1.189 \sim 1.735)$ & 0.001 & $1.395(1.153 \sim 1.688)$ & 0.001 & $1.395(1.153 \sim 1.687)$ & 0.001 \\
\hline pN classification & $1.448(1.288 \sim 1.615)$ & 0.000 & - & - & $1.050(0.869 \sim 1.268)$ & 0.613 \\
\hline rN classification & - & - & $1.646(1.449 \sim 1.870)$ & 0.000 & $1.579(1.284 \sim 1.941)$ & 0.000 \\
\hline
\end{tabular}

AEG, adenocarcinoma of the esophagogastric junction; RFS, recurrence-free survival; OS, overall survival;

$\mathrm{HR}$, hazard ratio; CI, confidence interval; $\mathrm{pN}$, lymph node status; rN, N-ratio.

classification and used for lymph node involvement, this factor (RFS: $P<0.001$; OS: $P<0.001$ ) was identified as an independent prognostic factor in addition to $\mathrm{pT}$ classification (RFS: $P<0.001$; OS: $P<0.001$ ). However, when both the $\mathrm{pN}$ and $\mathrm{rN}$ classification were combined in the model at the same time, rN classification was highly significant (RFS: $P<0.001$; OS: $P<0.001$ ), while $\mathrm{pN}$ classification was not a significant factor. These results are shown in Table 2.

We used the adjusted hazard ratio (HR) and $-2 \log$ likelihood to evaluate the discriminating abilities of each staging system. As a result, we found that the HRs for $\mathrm{rN}$ (RFS:1.637; OS:1.646) were larger than $\mathrm{pN}$ classification (RFS:1.442; OS:1.448). While the -2log likelihood for rN (RFS:3066.658; OS:2879.804) were smaller than $\mathrm{pN}$ classification (RFS:3087.341; OS:2899.995). Therefore, we inferred that $\mathrm{rN}$ classification may provide a better prognosis estimation than $\mathrm{pN}$ classification for $\mathrm{AEG}$ patients.

\section{Survival analysis based on pN classification according to the $\mathrm{rN}$ classification}

The 5-year RFS and OS rates of patients were compared with different $\mathrm{pN}$ when stratifying by $\mathrm{rN}$ classifications and with different $\mathrm{rN}$ when stratifying by pN classifications. As shown in Table 3, for RFS, there were significant differences in 5-year RFS rates among different $\mathrm{rN}$ classifications within the same $\mathrm{pN}(P<0.05)$, but not among different $\mathrm{pN}$ classifications within the same $\mathrm{rN}(P>0.05)$. For OS, there were significant differences in 5-year OS rates among different $\mathrm{rN}$ classifications within the same $\mathrm{pN}(P<0.05)$, but not among different $\mathrm{pN}$ classifications within the same $\mathrm{rN}(P>0.05)$.

\section{Analyses of the most appropriate prognostic classification for the survival prediction of AEG patients}

In the TNM staging system, the 5-year RFS rates of patients at stages I, II, IIIA, IIIB and IIIC were 52.8\%, $35.2 \%, 26.8 \%, 15.4 \%$ and $4.4 \%$, respectively $(P<0.001$, Figure $3 \mathrm{~A})$. The 5-years OS rates of patients at stages I, II, IIIA, IIIB and IIIC were $61.1 \%, 40.7 \%, 31.8 \%, 17.3 \%$ and $5.8 \%$, respectively $(P<0.001$, Figure $3 \mathrm{~B})$. In the modified TrNM staging system, the 5-year RFS rates of patients at stages I, II, IIIA, IIIB and IIIC were $50.0 \%$, $38.4 \%, 21.7 \%, 12.1 \%$ and $1.9 \%$, respectively $(P<0.001$, Figure $3 \mathrm{C}$ ). The 5 -years OS rates of patients at stages I, II, IIIA, IIIB and IIIC were $57.9 \%, 44.0 \%, 25.8 \%, 15.7 \%$ and $2.4 \%$, respectively $(P<0.001$, Figure $3 \mathrm{D})$. These results are listed in Table 4.

Moreover, the survival discriminatory ability between the TNM and TrNM staging systems was evaluated by the HR and $-2 \log$ likelihood. We also found that, compared with the 7th edition TNM staging system, the TrNM staging system was the most appropriate prognostic classification for predicting the RFS (HR: 1.647 vs.1.495; -2log likelihood: 3062.115 vs. 3078.584) and OS (HR: 1.664 vs.1.494; -2log likelihood: 2874.578 vs. 2892.807). 
Table 3: RFS and OS rates with different $\mathrm{pN}$ classifications stratified by the $\mathrm{rN}$ classification

\begin{tabular}{|c|c|c|c|c|c|c|c|c|c|c|c|}
\hline \multirow{2}{*}{$\begin{array}{l}\text { Lymph } \\
\text { classification }\end{array}$} & \multirow[b]{2}{*}{ node } & \multicolumn{2}{|l|}{ rNo } & \multicolumn{2}{|c|}{ rN1 } & \multicolumn{2}{|l|}{ rN2 } & \multicolumn{2}{|l|}{ rN3 } & \multirow[b]{2}{*}{$\chi^{2}$} & \multirow[b]{2}{*}{$P$} \\
\hline & & No. & \begin{tabular}{|l|} 
5-YSR \\
$(\%)$
\end{tabular} & No. & 5-YSR (\%) & No. & $\begin{array}{l}\text { 5-YSR } \\
(\%)\end{array}$ & No. & 5-YSR (\%) & & \\
\hline \multicolumn{12}{|l|}{ RFS } \\
\hline $\mathrm{pN} 0$ & & 135 & 40.7 & - & - & - & - & - & - & - & - \\
\hline pN1 & & - & - & 80 & $28.8 \%$ & 11 & $27.3 \%$ & 6 & 0 & 13.202 & 0.001 \\
\hline $\mathrm{pN} 2$ & & - & - & 40 & $27.5 \%$ & 33 & $12.1 \%$ & 11 & 0 & 7.500 & 0.024 \\
\hline $\mathrm{pN} 3$ & & - & - & 7 & 0 & 29 & $10.3 \%$ & 37 & $2.7 \%$ & 9.433 & 0.009 \\
\hline$\chi^{2}$ & & \multicolumn{2}{|l|}{-} & \multicolumn{2}{|c|}{1.520} & \multicolumn{2}{|c|}{1.264} & \multicolumn{2}{|c|}{0.287} & & \\
\hline$P$ & & \multicolumn{2}{|l|}{-} & \multicolumn{2}{|c|}{\begin{tabular}{|l|}
0.468 \\
\end{tabular}} & \multicolumn{2}{|c|}{0.532} & \multicolumn{2}{|c|}{0.762} & & \\
\hline \multicolumn{12}{|l|}{ OS } \\
\hline $\mathrm{pN} 0$ & & 135 & 47.0 & - & - & - & - & - & - & - & - \\
\hline $\mathrm{pN} 1$ & & - & - & 80 & $33.5 \%$ & 11 & $34.1 \%$ & 6 & 0 & 9.617 & 0.008 \\
\hline $\mathrm{pN} 2$ & & - & - & 40 & $29.6 \%$ & 33 & $15.2 \%$ & 11 & 0 & 9.139 & 0.010 \\
\hline $\mathrm{pN} 3$ & & - & - & 7 & 0 & 29 & $13.5 \%$ & 37 & $3.9 \%$ & 13.335 & 0.001 \\
\hline$\chi^{2}$ & & \multicolumn{2}{|l|}{-} & \multicolumn{2}{|c|}{\begin{tabular}{|l|}
2.508 \\
\end{tabular}} & \multicolumn{2}{|c|}{1.236} & \multicolumn{2}{|c|}{0.254} & & \\
\hline$P$ & & \multicolumn{2}{|l|}{-} & \multicolumn{2}{|c|}{0.285} & \multicolumn{2}{|c|}{0.539} & \multicolumn{2}{|c|}{0.881} & & \\
\hline
\end{tabular}

RFS, recurrence-free survival; OS, overall survival; 5-YSR, 5-year survival rate;

$\mathrm{pN}$, lymph node status; rN, N-ratio.

Table 4: RFS and OS rates according to the TNM and TrNM staging system in 389 AEG patients after curative surgery

\begin{tabular}{|c|c|c|c|c|c|c|c|c|c|}
\hline \multirow[b]{2}{*}{$\begin{array}{l}\text { Clinicopathologic } \\
\text { factors }\end{array}$} & \multirow[b]{2}{*}{ Cases } & \multicolumn{4}{|c|}{ Recurrence-free survival } & \multicolumn{4}{|c|}{ Overall survival } \\
\hline & & \begin{tabular}{|l|}
$5-Y S R$ \\
$(\%)$
\end{tabular} & $\chi^{2}$ & HR $(95 \% C I)$ & $P$ value & \begin{tabular}{|l|} 
5-YSR \\
$(\%)$
\end{tabular} & $\chi^{2}$ & HR $(95 \% C I)$ & $P$ value \\
\hline TNM staging system & & & 78.935 & $\begin{array}{l}1.495 \\
(1.361 \sim 1.643)\end{array}$ & 0.000 & & 71.957 & \begin{tabular}{|l}
1.494 \\
$(1.355 \sim 1.647)$
\end{tabular} & 0.000 \\
\hline I & 36 & 52.8 & & & & 61.1 & & & \\
\hline II & 125 & 35.2 & & & & 40.7 & & & \\
\hline IIIA & 82 & 26.8 & & & & 31.8 & & & \\
\hline IIIB & 78 & 15.4 & & & & 17.3 & & & \\
\hline IIIC & 68 & 4.4 & & & & 5.8 & & & \\
\hline TrNM staging system & & & 112.373 & $\begin{array}{l}1.647 \\
(1.484 \sim 1.829)\end{array}$ & 0.000 & & 111.396 & \begin{tabular}{|l|}
1.664 \\
$(1.492 \sim 1.855)$
\end{tabular} & 0.000 \\
\hline $\mathrm{I}$ & 38 & 50.0 & & & & 57.9 & & & \\
\hline II & 125 & 38.4 & & & & 44.0 & & & \\
\hline IIIA & 115 & 21.7 & & & & 25.8 & & & \\
\hline IIIB & 58 & 12.1 & & & & 15.7 & & & \\
\hline IIIC & 53 & 1.9 & & & & 2.4 & & & \\
\hline
\end{tabular}

AEG, adenocarcinoma of the esophagogastric junction; RFS, recurrence-free survival; OS, overall survival; 5-YSR, 5-year survival rate;

HR, hazard ratio; CI, confidence interval; TNM, tumor- node- metastasis; TrNM, tumor- node ratio- metastasis.

\section{Comparison of the predictive accuracy for the survival of patients with AEG}

Finally, the area under the receiver operating characteristic curve (AUC) and the Harrell's concordance index (C-index) values for each staging system were calculated to evaluate the predictive accuracy of the aforementioned staging systems.

For RFS, the AUC of the $\mathrm{rN}$ and $\mathrm{pN}$ classifications were $0.759,0.738$, respectively, the difference was not significantly different $(\mathrm{z}=0.582, P>0.05)$. The AUC for the TrNM and TNM staging system were $0.776,0.764$, respectively, there was also no significantly different $(\mathrm{z}=$ $0.293, P>0.05)$. Furthermore, for OS, the AUC of the rN, $\mathrm{pN}, \mathrm{TrNM}$ and TNM staging system were $0.736,0.721$, 0.756 and 0.739 , respectively, no significantly different was observed among these staging systems $(\mathrm{z}=0.400$, $0.454, P>0.05$, for all) (Table 5, Figure 4).

Similarly, for RFS, the rN and the modified TrNM staging system presented a significantly larger C-index compared with the $\mathrm{pN}$ and TNM staging system. Moreover, for OS, the $\mathrm{rN}$ and $\mathrm{TrNM}$ staging system yielded a significantly larger $\mathrm{C}$-index than the $\mathrm{pN}$ and TNM staging system did (Table 5). 
Table 5: Prognostic ability comparison among the different staging systems for patients with AEG

\begin{tabular}{|l|c|c|c|c|c|c|c|c|c|}
\hline \multirow{2}{*}{ Staging systems } & \multicolumn{4}{|c|}{ RFS } & \multicolumn{5}{c|}{ OS } \\
\cline { 2 - 10 } & HR & -2 log likelihood & AUC & C-index & HR & -2log likelihood & AUC & C-index \\
\hline rN classification & 1.637 & 3066.658 & 0.759 & 0.729 & 1.646 & 2879.804 & 0.736 & 0.714 \\
\hline pN classification & 1.442 & 3087.341 & 0.738 & 0.701 & 1.448 & 2899.995 & 0.721 & 0.697 \\
\hline TrNM staging system & 1.647 & 3062.115 & 0.776 & 0.745 & 1.664 & 2874.578 & 0.756 & 0.726 \\
\hline TNM staging system & 1.495 & 3078.584 & 0.764 & 0.722 & 1.494 & 2892.807 & 0.739 & 0.701 \\
\hline
\end{tabular}

AEG, adenocarcinoma of the esophagogastric junction; AUC, area under the curve; C-index: concordance index $\mathrm{pN}$, lymph node status; rN, N-ratio; TNM, tumor- ratio-metastasis; TrNM, tumor- node ratio-metastasis

These results indicated that the $\mathrm{rN}$ and $\operatorname{TrNM}$ staging system may have better prognostic stratification and more precise prediction than the $\mathrm{pN}$ and TNM staging system for patients with AEG.
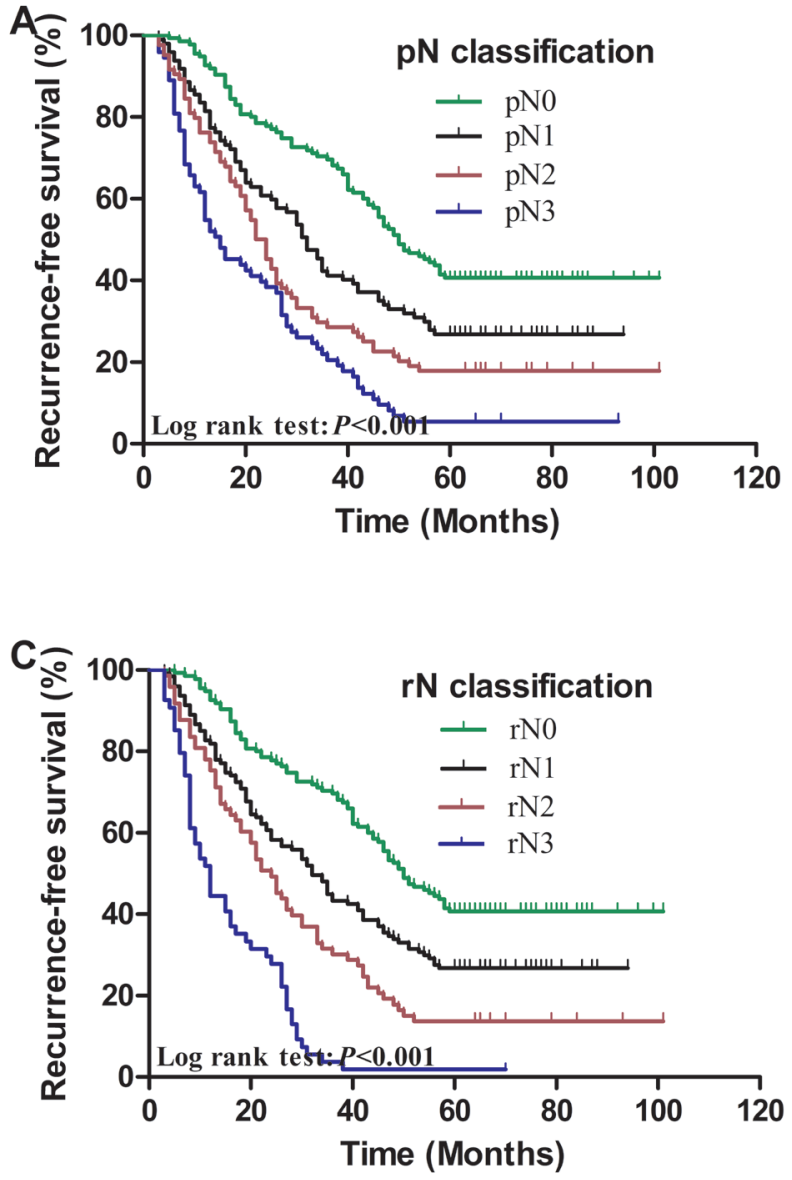

\section{DISCUSSION}

An effective tumor staging system is used to predict prognosis and determine the most appropriate multidisciplinary therapeutic modalities. These objectives can be accomplished by applying a feasible, reproducible, and accurate staging system for the prognostic stratification without stage migration of AEG.
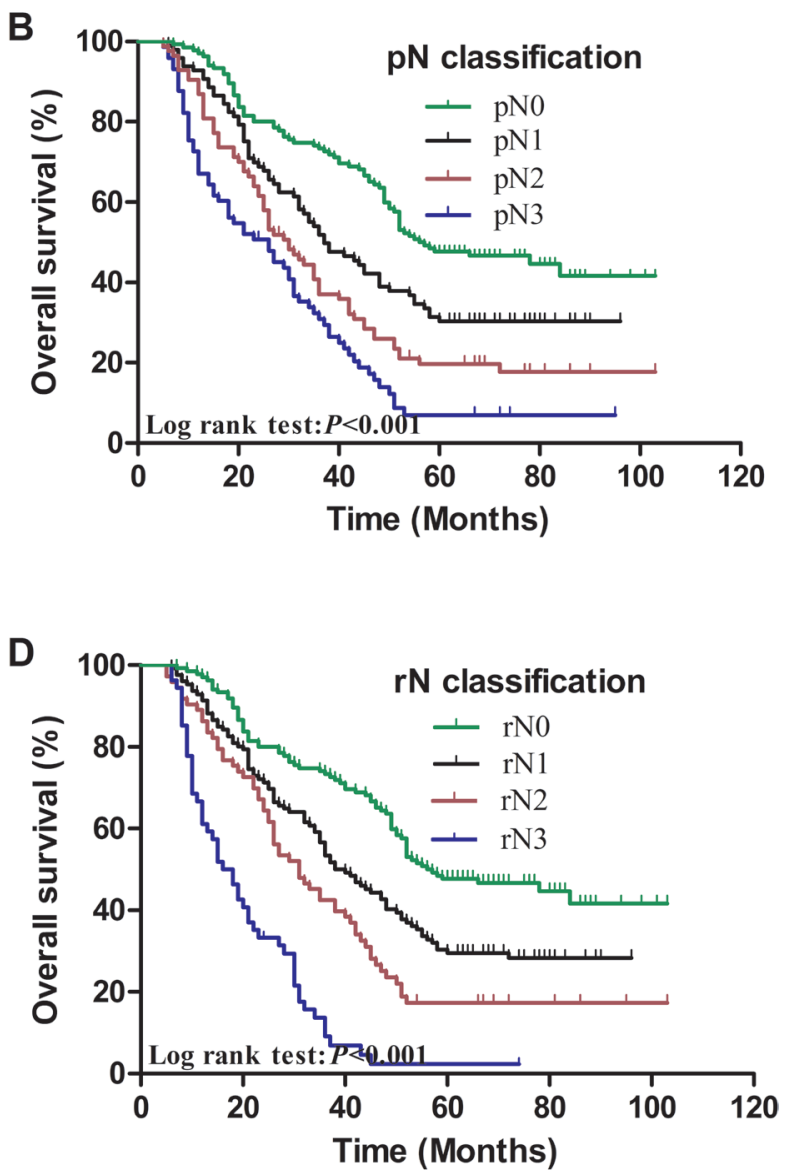

Figure 2: Kaplan-Meier survival curves of recurrence-free survival and overall survival in patients with AEG according to $\mathbf{p N}$ and $\mathbf{r N}$ classifications. A., B., effect of $\mathrm{pN}$ classification; C., D., effect of $\mathrm{rN}$ classification $(P$-values were calculated by the log-rank test). 
The presence of lymph node metastasis has been previously considered as an important prognostic factor, and it has been incorporated into the AJCC TNM staging system [9, 10]. However, in this staging system, the number of metastatic LNs is influenced by the number of removed LNs, which itself depends on several controllable factors, such as surgical procedures or pathological evaluation; and some uncontrollable factors, such as patient and tumor characteristics. In cases few LNs are removed, the $\mathrm{N}$ stage cannot be classified accurately. Thus, incorrect pathologic staging would occur and lead to improper treatment. This phenomenon is well-known as stage migration or Will Roger's phenomenon [11].

Although curative resection remains as the cornerstone for the treatment of AEG, the appropriate procedures of surgery, extent of lymphadenectomy and the exact number of LNs should be examined remain unaddressed for this type of tumor; this limitation is attributed to its dependence on tumor location and safety margin length achieving $\mathrm{R} 0$ resection $[3,9,12]$. Van Cutsem [13] and Barbour et al [14] suggested that patients with Siewert types II and III AEG should undergo
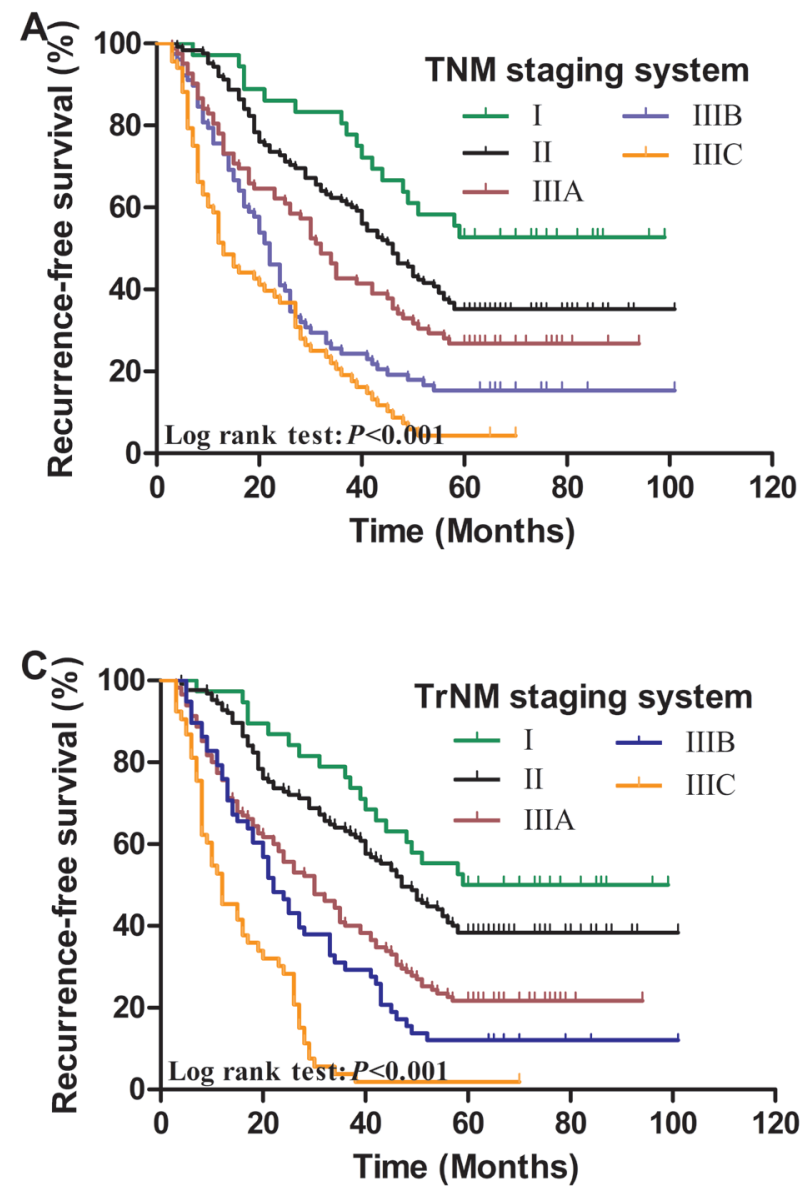

adequate lymphadenectomy with the removal of $\geq 15$ LNs to assess the $\mathrm{pN}$ stage precisely. Conducting a large retrospective study, Gee et al [15] stated that preferably 20-25 LNs should be removed to determine the prognosis and treatment for AEG tumors.

The use of $\mathrm{rN}$, which is defined as the ratio between metastatic and examined LNs, has been proposed by several authors. It has been deemed to be a simple, reliable, and reproducible method that can be used to better evaluate the status of lymph node metastasis and to identify the subgroup of patients with esophageal, gastric, breast, and colon cancer with similar prognosis; thus minimizing the "stage migration" phenomenon that can be observed when the conventional TNM staging system is used $[16,17]$. Furthermore, it is applicable to cases with insufficient number of retrieved LNs [7, 18, 19] or to cases subjected to limited lymphadenectomy [20, 21].

We found that $\mathrm{rN}$ was correlated with the number of metastatic nodes but not with the total number of removed LNs. This finding indicated that a specific number of dissected LNs are required to ensure an accurate assessment of lymph node status. Using rN may
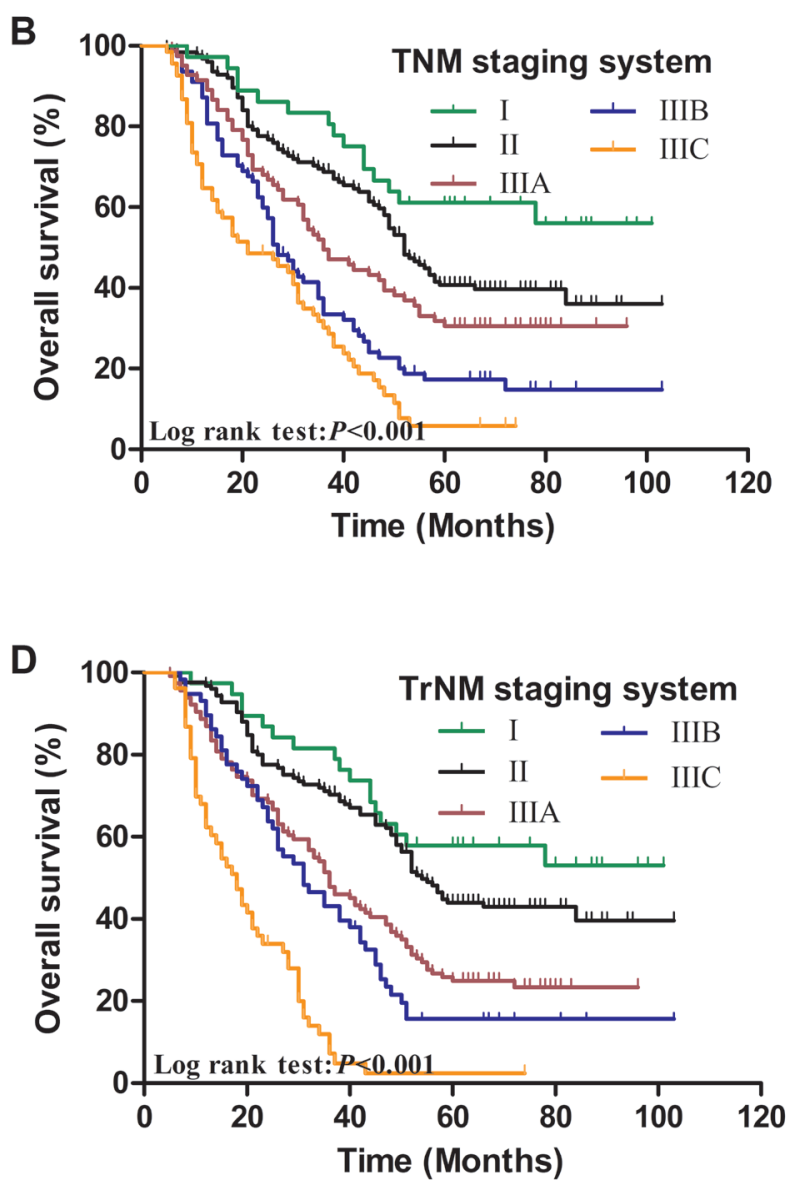

Figure 3: Kaplan-Meier survival curves of recurrence-free survival and overall survival in patients with AEG according to TNM and TrNM staging systems. A., B., effect of TNM staging system; C., D., effect of TrNM staging system $(P$-values were calculated by the log-rank test). 
significantly reduce the potential bias and minimize stage migration phenomenon because it is slightly influenced by the type of lymphadenectomy and the number of dissected LNs.

However, up until now, there is still no consensus on the optimal categorization with regard to the $\mathrm{rN}$ has been reported in previous literatures and the most suitable methods to determine cut-off levels also differ among studies $[4,7,18,19,22]$. Liu et al. [19] classified 1,325 esophageal cancer patients into four groups based on $\mathrm{rN}: 0,0-0.25,0.25-0.5$, and $>0.5$, and confirmed that an increasing $\mathrm{rN}$ was linearly associated with a poorer long-term survival than $\mathrm{pN}$ category when an insufficient number of LNs were examined. According to a retrospective study involving a series of 710 patients with gastric cancer, the ratio was classified as $0,1 \%$ $10 \%, 10 \%-25 \%$, and $>25 \%$. They demonstrated that the $\mathrm{N}$-ratio was a reliable classification that may improve the current nodal staging system and help stratify prognosis regardless of the number of examined LNs [18]. In a study [23] using the database of the Surveillance, Epidemiology and End Results (SEER) data of 18,043 gastric cancer patients subjected to gastrectomy, $\mathrm{rN}$ ratio was identified as follows: rN1: $0-1 / 15, \mathrm{rN} 2: 1 / 15-3 / 10, \mathrm{rN} 3: 3 / 10-$ 7/10, and rN 4: > 7/10; a TrNM staging system was also constructed. The misclassification rate is $12 \%$ for the

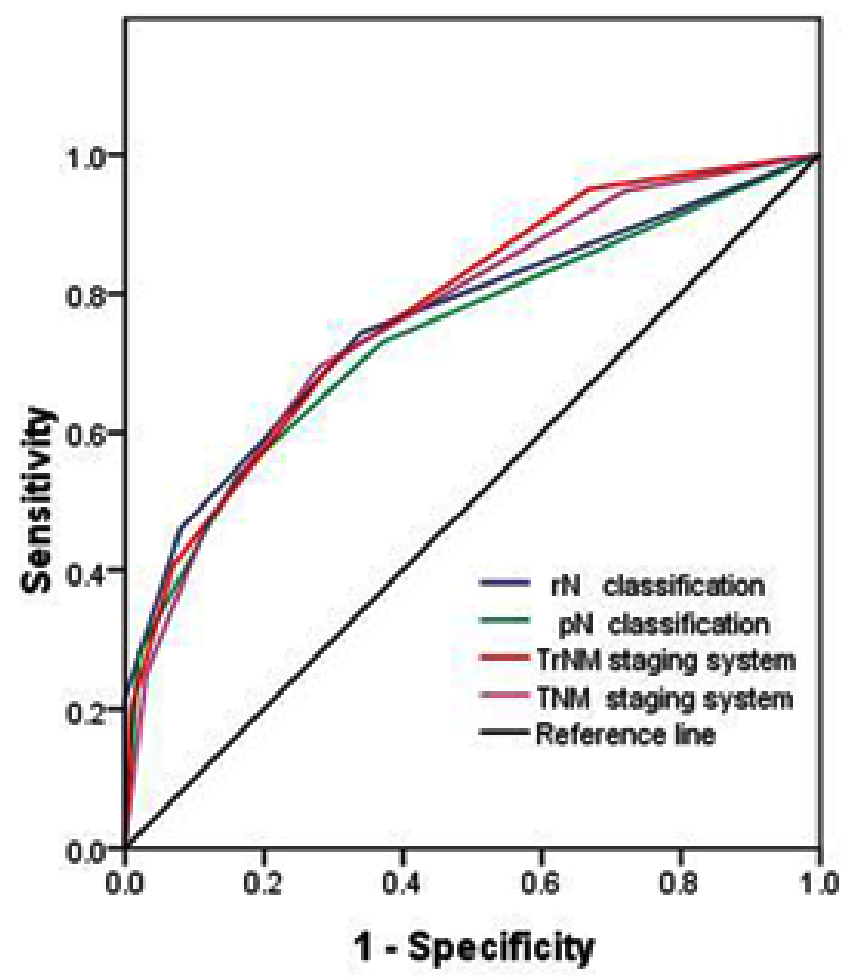

A proposed TrNM system. Therefore, the modified TNrM system is more effective and rational than the current TNM system in the staging of gastric cancer in the SEER database. The system can also help doctors determine the patients who can benefit from adjuvant treatments.

In the present study, the ratio was classified by the best cut-off approach as follows: $0,1-30 \%, 31-60 \%$, and $>60 \%$. Kaplan-Meier survival analysis revealed that, both $\mathrm{pN}$ and $\mathrm{rN}$ classifications were significantly associated with RFS and OS. Subsequent multistep multivariate analysis indicated that, either $\mathrm{pN}$ or $\mathrm{rN}$ can be used as an independent variable for RFS and OS. However, rN remained as an independent prognostic factor and $\mathrm{pN}$ lost its significance when both $\mathrm{pN}$ and $\mathrm{rN}$ categories were introduced as covariates to the same model. This phenomenon is consistent with that reported by other investigators. Furthermore, the subgroups of AEG patients can be identified in more detail and more precision by using the $\mathrm{rN}$ category than the $\mathrm{N}$ category.

In addition, on the basis of the superiority of $\mathrm{rN}$ classification to $\mathrm{pN}$ classification, we therefore combined the $\mathrm{pT}$ and $\mathrm{rN}$ classification to form the hypothetical TrNM staging system and then compared it with the conventional TNM staging system. The survival rate could be easily distinguished between patients when the $\mathrm{N}$ classifications were replaced with $\mathrm{rN}$ classifications in

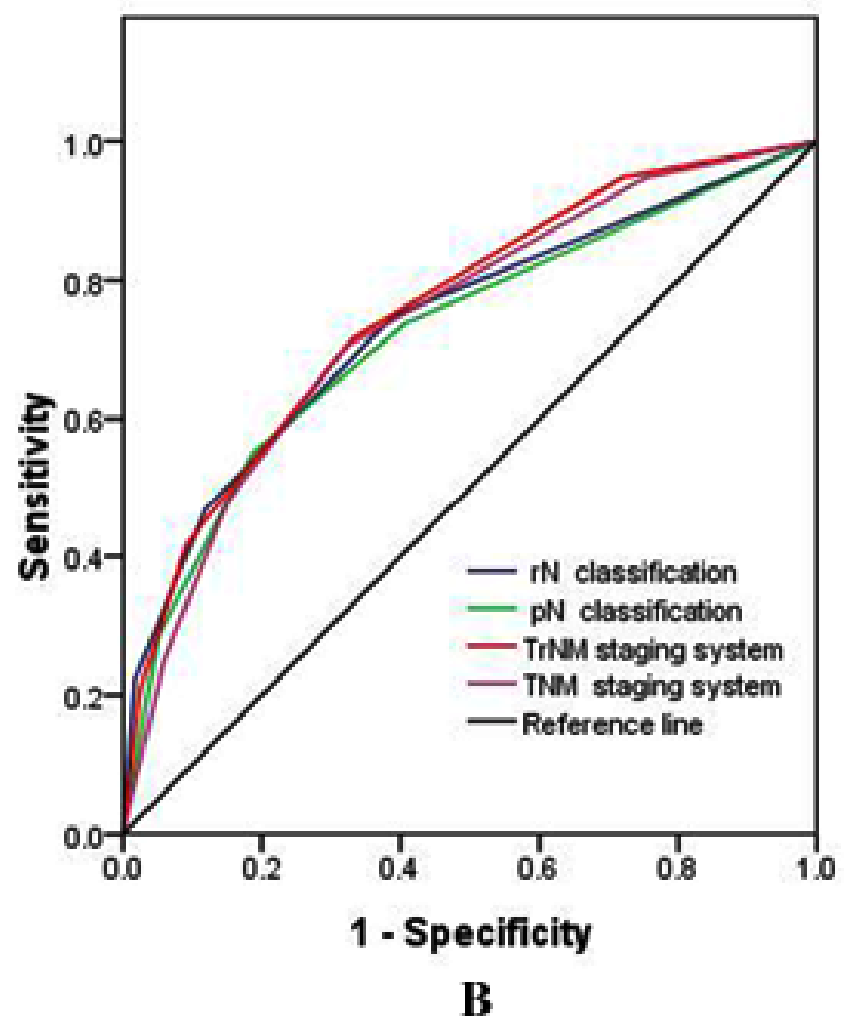

Figure 4: Receiver operating characteristic curves of the $\mathrm{pN}$ and $\mathrm{rN}$ classifications, TNM and TrNM staging systems for the prediction of the survival of AEG patients after curative surgery. A., For RFS; and B., For OS. 
the staging system. Comparing the $-2 \log$ likelihood and $\mathrm{HR}$, we found that the proposed TrNM staging exhibited greater discriminatory abilities to predict survival than the traditional TNM staging did. In the predictive accuracy analysis, the most commonly used method is the AUC by ROC analysis [24]. In our study, the AUCs were larger in the $\mathrm{rN}$ and TrNM staging system than in the $\mathrm{pN}$ and TNM staging system in terms of RFS and OS. Moreover, the $\mathrm{rN}$ and TrNM staging system yielded a significantly larger $\mathrm{C}$-index than the $\mathrm{pN}$ and TNM staging system did. These findings demonstrated that $\mathrm{rN}$ should be recommended as an important variable to enhance the accuracy of the prognostic prediction of AEG patients.

The limitation of the current study is its retrospective analysis setting, including a relatively small sample size from a single center was included in the study. Therefore, a multiple-center clinical trial with a large sample size should prospectively provide further evidence to evaluate the optimal cut-off point of N-ratio. Moreover, the pathological diagnosis of lymph node metastasis was simply analyzed by hematoxylin and eosin staining. No special techniques, such as immunohistochemistry or reverse-transcription polymerase chain reaction, were routinely used to identify micrometastases [25]. In addition, almost half of the patients unlikely gain the benefits of the $\mathrm{rN}$ classification for predicting outcomes because the definition of the rN0 classification is consistent with the pN0 classification. Log odds of positive LNs, which is defined as the log of the ratio between the number of positive and negative LNs, has been proposed recently $[26,27]$. However, the strength of this study is that we did not limit our analysis to the OS but also included the RFS, which is a specific endpoint in the field of cancerrelated investigations. The adoption of this new TrNM staging system may help oncologists predict prognosis, make sound treatment decisions, and facilitate informed discussion with patients in terms of recurrence and death, especially for those who underwent limited lymph node dissection.

In conclusion, we demonstrated that the $\mathrm{rN}$ classification is a potentially useful and reliable factor to predict RFS and OS for patients with AEG. Incorporation of $\mathrm{rN}$ into the current staging system could help address the limitations of $\mathrm{N}$ classification and enable clinicians to predict the prognosis of AEG patients accurately. Nevertheless, further studies should be conducted to overcome the limitations of our study and validate our results.

\section{PATIENTS AND METHODS}

\section{Study design and patient population}

From January 2000 to January 2007, a retrospective database of 389 patients with histologically confirmed adenocarcinoma of the gastroesophageal junction (AEG II-III), who had underwent curative resection and systematic lymphadenectomy in the thoracic surgery and gastrointestinal surgery departments were retrospectively reviewed. This study protocol was approved by the Research Ethics Committee of Tianjin Medical University Cancer Institute and all patients were provided written informed consent for the use of their information in the hospital database.

The eligibility criteria included histologically confirmed R0 resection, which was defined as no macroscopic and microscopic residual tumor and a postoperative survival time of $>3$ months. None of these cases received any kind of neoadjuvant treatments, such as preoperative chemotherapy and/or radiotherapy. Type I lesions of the gastroesophageal junction were designated as cancers of the esophagus; and patients with distant metastasis or peritoneal dissemination confirmed during the surgery were excluded from the study. Based on these criteria, 389 patients with types II and III tumors were included in the study. Their demographic and clinical characteristics are shown in Table 1.

Before surgery was performed, endoscopy and barium swallows were performed to determine Siewert type. Chest radiography, cervical and abdominal ultrasonography, and computed tomography (CT) scans from the neck to the upper abdomen were conducted to exclude liver and lung metastasis. Bone scanning was selectively carried out to exclude bone metastasis for patients with symptoms of bone pain. The operation was selected based on preoperative diagnosis and estimated length of esophageal invasion. All of the patients underwent a proximal or total gastrectomy with lymphadenectomy via a transthoracic or transabdominal approach.

The resected specimens were histologically examined by the same group of gastrointestinal pathologists in our institution. Histopathological types were classified as differentiated (well/moderately differentiated or papillary adenocarcinoma) or undifferentiated (poorly differentiated or undifferentiated adenocarcinoma, signet-ring cell carcinoma or mucinous adenocarcinoma or other types of tumors) according to the World Health Organization (WHO) classifications [28]. According to Lauren's classification, the cancers were classified as intestinal type and diffuse-mixed type [29]. All patients were staged on the basis of the 7th edition of the American Joint Committee on Cancer (AJCC) 
TNM staging system for esophagus and esophagogastric junction cancer $[10,30]$.

\section{Lymph node classification}

Lymph node metastasis was classified according to the 7th edition AJCC N staging system: pN0 refers to no metastasis; $\mathrm{pN} 1$ refers to $1 \sim 2$ positive $\mathrm{LNs}$; $\mathrm{pN} 2$ refers to 3 6 positive LNs; and $\mathrm{pN} 3$ refers to 7 or more positive LNs.

The N-ratio was calculated as the ratio of the number of metastatic LNs to the total number of removed LNs. rN intervals was determined by the best cut-off approach in terms of the log-rank test. To make the study compatible with the 7th edition of AJCC TNM staging system, we proposed a novel TrNM hypothetical staging system on the basis of the pT classification and the aforementioned $\mathrm{rN}$ classifications. The TrNM staging system is as follows: I (IA, T1rN0; IB, T1rN1, T2rN0); II (IIA, T1rN2, T2rN1, T3rN0; IIB, T1rN3, T2rN2, T3rN1,T4arN0); IIIA(T2rN3, T3rN2, T4arN1); IIIB(T3rN3, T4arN2, T4brN0, T4brN1); IIIC(T4arN3, T4brN2, T4brN3). Then, RFS and OS rates based on $\mathrm{pN}$ and $\mathrm{rN}$ as well as the TNM, TrNM staging were compared.

\section{Follow-up}

After curative resection, all patients were generally monitored every 3 months for the first 2 years, every 6 months during the third to fifth years, and then every year until death or the last follow-up. Check-up items included physical examination, tumor-marker examination, chest radiography, CT scans, neck and abdominal ultrasonography, endoscopic examination, and bone scan when necessary to detect recurrence and/ or metastasis. Recurrence of the disease was determined by clinical examination or imaging method. The follow up was completed in September 2012 with a rate of $92.3 \%$ and the median follow-up period was 36 months (range, 3 144 months). The cases lost to follow-up were treated as censored data for the analysis of survival rates.

The primary endpoint of the study was recurrencefree survival (RFS), which was defined as the period from surgery to disease progression or recurrence or the date of death or last follow-up. The secondary endpoint was overall survival (OS), which was calculated as the time from operation to the point of death or last follow-up.

\section{Statistical analysis}

Statistical analyses were performed using SPSS 17.0 (SPSS, Inc., Chicago, IL) software and programming languages R (version 3.2.2 for Windows). Spearman correlation analysis was calculated to assess the correlation among the number of retrieved LNs, metastatic LNs and $\mathrm{rN}$. The rN cut-off was determined by the best cut-off approach using log-rank test as previous study reported $[18,27]$. The 5-year RFS, OS analyses were calculated on the basis of the Kaplan-Meier method and compared by the log-rank test. Factors deemed as potentially important by univariate analyses $(P<0.05)$ were subjected to multivariate Cox proportional hazards model to identify the independent prognostic factors. Multivariate survival analyses were performed using the Cox regression proportional hazards model to identify the independent factors. The HR and -2log likelihood values within a Cox regression were calculated for each category to measure its discriminatory ability. The larger the HR, the better the system. While the smaller the -2log likelihood, the better the system. Furthermore, the predictive accuracy of different categories was evaluated using the area under the receiver operating characteristic curve (AUC) and the Harrell's concordance index (C-index). The larger the AUC or C-index, the more precise was the survival prediction [31]. A two-tailed $P$ value of $<0.05$ was considered to be statistically significant..

\section{ACKNOWLEDGMENTS}

This work was supported by the National Key Clinical Specialist Construction Programs of China(NO.2013-544), the National Natural Science Foundation of China (81071981), Key grant of health and family planning commission of Tianjin(14KG143) and Tianjin City High School Science \& Technology Fund Planning Project(No. 20130121).

\section{CONFLICTS OF INTEREST}

The authors declare no conflict of interest.

\section{REFERENCES}

1. Amini N, Spolverato G, Kim Y, Squires MH, Poultsides GA, Fields R, Schmidt C, Weber SM, Votanopoulos K, Maithel SK, Pawlik TM. Clinicopathological features and prognosis of gastric cardia adenocarcinoma: a multiinstitutional US study. J Surg Oncol. 2015;111:285-292.

2. Hosoda K, Yamashita K, Katada N, Watanabe M. Overview of multimodal therapy for adenocarcinoma of the esophagogastric junction. Gen Thorac Cardiovasc Surg. 2015;63:549-556.

3. Sisic L, Blank S, Weichert W, Jager D, Springfeld C, Hochreiter M, Buchler M, Ott K. Prognostic impact of lymph node involvement and the extent of lymphadenectomy (LAD) in adenocarcinoma of the esophagogastric junction (AEG). Langenbecks Arch Surg. 2013;398:973-981. 
4. Tan Z, Ma G, Yang H, Zhang L, Rong T, Lin P. Can lymph node ratio replace pn categories in the tumor-nodemetastasis classification system for esophageal cancer? J Thorac Oncol. 2014;9:1214-1221.

5. Barbour AP, Rizk NP, Gonen M, Tang L, Bains MS, Rusch VW, Coit DG, Brennan MF. Lymphadenectomy for adenocarcinoma of the gastroesophageal junction (GEJ): impact of adequate staging on outcome. Ann Surg Oncol. 2007; 14:306-316.

6. Wu XJ, Miao RL, Li ZY, Bu ZD, Zhang LH, Wu AW, Zong XL, Li SX, Shan F, Ji X, Ren H, Ji JF. Prognostic value of metastatic lymph node ratio as an additional tool to the TNM stage system in gastric cancer. Eur J Surg Oncol. 2015;41:927-933.

7. Wei C, Deng WY, Li N, Shen W, Zhang C, Liu JY, Luo SX. Lymph Node Ratio as an Alternative to the Number of Metastatic Lymph Nodes for the Prediction of Esophageal Carcinoma Patient Survival. Dig Dis Sci. 2015;60:27712776.

8. Zhao LY, Li CC, Jia LY, Chen XL, Zhang WH, Chen XZ, Yang K, Liu K, Wang YG, Xue L, Zhang B, Chen ZX, Chen JP, Zhou ZG, Hu JK. Superiority of lymph node ratio-based staging system for prognostic prediction in 2575 patients with gastric cancer: validation analysis in a large single center. Oncotarget. 2016; doi: 10.18632/ oncotarget.9714.

9. Peng J, Wang WP, Yuan Y, Hu Y, Wang Y, Chen LQ. Optimal Extent of Lymph Node Dissection for Siewert Type II Esophagogastric Junction Adenocarcinoma. Ann Thorac Surg. 2015;100:263-269.

10. Yoshikawa T. [Esophagogastric junction cancer in the TNM classification]. [Article in Japanese]. Nihon Geka Gakkai Zasshi. 2015;116:35-39.

11. Feinstein AR, Sosin DM, Wells CK. The Will Rogers phenomenon. Stage migration and new diagnostic techniques as a source of misleading statistics for survival in cancer. N Engl J Med. 1985;312:1604-1608.

12. Zhang H, Wang W, Cheng Y, Song Y, Zhu K, Dang C. Adenocarcinomas of the esophagogastric junction: experiences at a single institution in China. World J Surg Oncol. 2013;11:155.

13. Van Cutsem E, Van de Velde C, Roth A, Lordick F, Kohne $\mathrm{CH}$, Cascinu S, Aapro M. Expert opinion on management of gastric and gastro-oesophageal junction adenocarcinoma on behalf of the European Organisation for Research and Treatment of Cancer (EORTC)-gastrointestinal cancer group. Eur J Cancer. 2008;44:182-194.

14. Barbour AP, Rizk NP, Gonen M, Tang L, Bains MS, Rusch VW, Coit DG, Brennan MF. Lymphadenectomy for adenocarcinoma of the gastroesophageal junction (GEJ): impact of adequate staging on outcome. Ann Surg Oncol. 2007;14:306-316.

15. Gee DW, Rattner DW. Management of localized esophageal cancer in the older patient. Oncologist. 2007;12:175-185.
16. Chen S, Zhao BW, Li YF, Feng XY, Sun XW, Li W, Zhou ZW, Zhan YQ, Qian CN, Chen YB. The prognostic value of harvested lymph nodes and the metastatic lymph node ratio for gastric cancer patients: results of a study of 1,101 patients. PLoS One. 2012;7:e49424.

17. Chen SB, Weng HR, Wang G, Zou XF, Liu DT, Chen YP, Zhang H. Lymph node ratio-based staging system for esophageal squamous cell carcinoma. World J Gastroenterol. 2015;21:7514-7521.

18. Alatengbaolide, Lin D, Li Y, Xu H, Chen J, Wang B, Liu C, $\mathrm{Lu}$ P. Lymph node ratio is an independent prognostic factor in gastric cancer after curative resection (R0) regardless of the examined number of lymph nodes. Am J Clin Oncol. 2013;36:325-330.

19. Liu YP, Ma L, Wang SJ, Chen YN, Wu GX, Han M, Wang $\mathrm{XL}$. Prognostic value of lymph node metastases and lymph node ratio in esophageal squamous cell carcinoma. Eur J Surg Oncol. 2010;36:155-159.

20. Pedrazzani C, Sivins A, Ancans G, Marrelli D, Corso G, Krumins V, Roviello F, Leja M. Ratio between metastatic and examined lymph nodes ( $\mathrm{N}$ ratio) may have low clinical utility in gastric cancer patients treated by limited lymphadenectomy: results from a single-center experience of 526 patients. World J Surg. 2010;34:85-91.

21. Maduekwe UN, Lauwers GY, Fernandez-Del-Castillo C, Berger DL, Ferguson CM, Rattner DW, Yoon SS. New metastatic lymph node ratio system reduces stage migration in patients undergoing D1 lymphadenectomy for gastric adenocarcinoma. Ann Surg Oncol. 2010;17:1267-1277.

22. Lee SR, Kim HO, Son BH, Shin JH, Yoo CH. Prognostic significance of the metastatic lymph node ratio in patients with gastric cancer. World J Surg. 2012;36:1096-1101.

23. Wang J, Dang P, Raut CP, Pandalai PK, Maduekwe UN, Rattner DW, Lauwers GY, Yoon SS. Comparison of a lymph node ratio-based staging system with the 7th AJCC system for gastric cancer: analysis of 18,043 patients from the SEER database. Ann Surg. 2012;255:478-485.

24. Li XQ, He WP, Hou WH, Chen JW, Fan RR, Yuan LJ, Yang GP, Cai MY, Chen L, Li J, He SY, Xie D, Yang GF, You ZS. Overexpression of RNF2 is positively associated with ovarian carcinoma aggressiveness and indicative of poor patient survival. Oncotarget. 2016; doi: 10.18632/ oncotarget.8975.

25. Wlodarczyk J, Mueller J, Wlodarczyk J. Lymph node micrometastases of adenocarcinoma located in gastroesophagal junction. Pol J Pathol. 2013;64:170-174.

26. Wen J, Ye F, He X, Li S, Huang X, Xiao X, Xie X. Development and validation of a prognostic nomogram based on the log odds of positive lymph nodes (LODDS) for breast cancer. Oncotarget. 2016;7:21046-21053. doi: 10.18632/oncotarget.8091.

27. Wu SG, Sun JY, Yang LC, Zhou J, Li FY, Li Q, Lin HX, Lin Q, He ZY. Prognosis of patients with esophageal squamous cell carcinoma after esophagectomy using the log 
odds of positive lymph nodes. Oncotarget. 2015;6:3691136922. doi: 10.18632/oncotarget.5366.

28. Flejou JF. [WHO Classification of digestive tumors: the fourth edition]. Ann Pathol. 2011;31:S27-S31.

29. Chen YC, Fang WL, Wang RF, Liu CA, Yang MH, Lo SS, Wu CW, Li AF, Shyr YM, Huang KH. Clinicopathological Variation of Lauren Classification in Gastric Cancer. Pathol Oncol Res. 2016;22:197-202.

30. Suh YS, Han DS, Kong SH, Lee HJ, Kim YT, Kim WH, Lee KU, Yang HK. Should adenocarcinoma of the esophagogastric junction be classified as esophageal cancer? A comparative analysis according to the seventh AJCC TNM classification. Ann Surg. 2012;255:908-915.
31. Fan H, Shao ZY, Xiao YY, Xie ZH, Chen W, Xie H, Qin GY, Zhao NQ. Comparison of the Glasgow Prognostic Score (GPS) and the modified Glasgow Prognostic Score (mGPS) in evaluating the prognosis of patients with operable and inoperable non-small cell lung cancer. J Cancer Res Clin Oncol. 2016;142:1285-1297. 